\title{
ON THE BEHAVIOR OF MEROMORPHIC FUNCTIONS AT THE IDEAL BOUNDARY OF A RIEMANN SURFACE
}

\author{
J. L. SCHIFF
}

\begin{abstract}
In a former work the author established an analog of a classical theorem of Painleve in the context of an arbitrary resolutive compactification of a Riemann surface. In the same setting, a refinement of the argument used in the above yields an elementary proof of a theorem of Riesz-LuzinPrivaloff type: If a meromorphic function $f$ tends to zero at each point of a subset $E$ of the ideal boundary and $E$ has positive harmonic measure, then $f \equiv 0$ on $R$. The well-known inclusion relations $U_{H B} \subset \theta_{M B^{*}}$, and $U_{H D^{-}}$ $\subset \vartheta_{M D^{*}}$, are then established from the point of view of the resolutivity of the Wiener and Royden compactification respectively.
\end{abstract}

1. Introduction. The boundary behavior of analytic and meromorphic functions has been a subject of investigation by mathematicians for more than three-quarters of a century. Early classical developments were due to Painlevé, Fatou, F. and M. Riesz, Luzin-Privaloff, Plessner, and Nevanlinna, with the theory being subsequently developed by numerous other authors. The unit disk, $|z|<1$, has been the domain of consideration in most of these works.

The ideal boundary behavior of a meromorphic function on a Riemann surface, which is the subject of this note, has been studied by Constantinescu and Cornea [1], and Sario and Nakai [4], amongst others. In the next section, we give a proof of a theorem of Riesz-Luzin-Privaloff type for an arbitrary resolutive compactification of a Riemann surface. The method of proof is both elemental in nature and fundamentally different from those given in the two aforementioned works. The Theorem is then used to establish a single direct line of proof of the well-known inclusion relations $U_{H B} \subset \vartheta_{M B^{*}}$, and $U_{H D^{-}}$ $\subset \mathfrak{C}_{M D^{*}}$

2. Preliminaries. Let $R^{*}$ be a resolutive compactification of a Riemann surface $R$, and set $\Delta=R^{*}-R$. The harmonic measure on $\Delta$ we denote by $\omega^{R^{*}}=\omega$. We quote the following result [2, Hilfssatz 8.8] as it is instrumental to our proof of the Main Theorem.

LEMMA . Let $R^{*}$ be a resolutive compactification and $G$ an open subset of $R$. If $s$ is a positive superharmonic function on $G$, then the set

\section{has harmonic measure zero.}

$$
A=\left\{\zeta \in \Delta-\overline{R-G}: \lim _{R \ni z \rightarrow \zeta} s(z)=\infty\right\}
$$

Received by the editors November 5, 1974.

AMS (MOS) subject classifications (1970). Primary 30A50, 30A48.

Key words and phrases. Resolutive compactification, ideal boundary, meromorphic function, superharmonic function, harmonic measure. 
3. Main results. We present here an elementary proof of a theorem of Riesz-Luzin-Privaloff type (cf. Constantinescu and Cornea [1], Sario and Nakai [4], Schiff [5]).

THEOREM. Let $R^{*}$ be a resolutive compactification of a Riemann surface $R$, and f a meromorphic function on $R$ such that $\lim _{R \ni z \rightarrow \zeta} f(z)=0$, for all $\zeta \in E \subset \Delta$. If $E$ has positive harmonic measure, then $f \equiv 0$ on $R$.

Proof. Suppose $f \not \equiv 0$ on $R$ and let $R^{\prime}=R-f^{-1}(\infty)$. Then $R^{\prime}$ is open in $R$, and the function $s=-\log |f|$ is superharmonic on $R^{\prime}$. Hence the set $G=\left\{z \in R^{\prime}: s(z)>0\right\}$ is an open subset of $R^{\prime}$, and a fortiori $G$ is open in $R$. The hypotheses of the Lemma are now satisfied, so that $\omega(A)=0$, where $A$ is defined in (1). For any $\zeta \in E \subset \Delta$, if $\zeta \notin A$, then $\lim _{R \ni z \rightarrow \zeta} s(z)=\infty$ implies $\zeta \in \overline{R-G}$. Hence there exists a net $\left\{z_{\alpha}\right\} \subset R-G$ such that $z_{\alpha} \rightarrow \zeta$. However, $s\left(z_{\alpha}\right) \leqslant 0$ (even if $z_{\alpha} \in f^{-1}(\infty)$ ), which violates the fact that $\lim _{z_{\alpha} \rightarrow \zeta} s\left(z_{\alpha}\right)=\infty$. It follows that $E \subset A$ and $\omega(E)=0$, contradicting $\omega(E)$ $>0$. We conclude that $f \equiv 0$ on $R$.

From the proof of the Theorem we obtain the following result:

COROLlaRY 1. If there exists a superharmonic function $s$ on $R$ such that $s \rightarrow \infty$ at each point of $E \subset \Delta$, then $\omega(E)=0$.

Whether or not the converse of this result is valid is an open question, however, it is well known to be false if we require $s$ to be positive.

In passing, we remark that if $p \in \Delta$ with $\omega(p)>0$, then it follows from the Theorem that any meromorphic function $f$ on $R$ with a continuous extension to $p$ satisfies $f(p) \neq \pm \infty$.

Let $U_{X}$ denote the class of Riemann surfaces which carry at least one $X$ minimal function for $X=H B, H D^{\sim}$. Here, $H B$ represents the class of bounded harmonic functions, and $H D^{\sim}$ the class of limits of nonincreasing sequences of positive Dirichlet-finite harmonic functions on $R$ which converge uniformly on compact subsets of $R$. Moreover, denote by $\hat{O}_{X}$ the null classes of Riemann surfaces for $X=M B^{*}, M D^{*}$, where $M B^{*}$ is the class of Lindelöfian meromorphic functions, and $M D^{*}$ the class of meromorphic functions with finite spherical Dirichlet integral (see Sario and Nakai [4] for a comprehensive account of these classes).

Corollary 2 (Constantinescu and Cornea [1], Kuramochi [3]). $U_{H B}$ $\subset \Theta_{M B^{*}}$

Proof. If $R \in U_{H B}$, it is known that the Wiener harmonic boundary contains a point $p$ of positive harmonic measure (cf. e.g. [4]). Since the Wiener compactification $R^{N}$ is resolutive, and every $f \in M B^{*}(R)$ has a continuous extension to $R^{N}$, the meromorphic function $f-f(p)$ vanishes at $p$. It follows from the Theorem that $f \equiv$ constant, and $R \in \mathcal{O}_{M B^{*}}$.

Corollary 3 (cF. Sario-Nakai [4]). $U_{H D} \sim \subset \mathcal{O}_{M D^{*}}$.

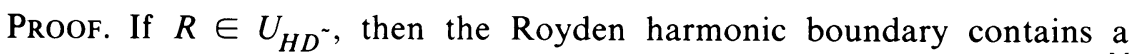
point $p$ of positive harmonic measure. Since the Royden compactification $R^{M}$ 
is resolutive and every $f \in M D^{*}(R)$ has a continuous extension to $R^{M}$, the meromorphic function $f-f(p)$ vanishes continuously at $p$. As above, $f \equiv$ constant, and $R \in O_{M D^{*}}$.

\section{REFERENCES}

1. C. Constantinescu and A. Cornea, Uber den idealen Rand und einige seiner Anwendungen bei der Klassifikation der Riemannschen Flächen, Nagoya Math. J. 13 (1958), 169-233, MR 20 \#3273.

2. - Ideale Ränder Riemannscher Flächen, Ergebnisse der Mathematik und ihrer Grenzgebiete, N. F., Band 32, Springer-Verlag, Berlin, 1963. MR $28 \# 3151$.

3. Z. Kuramochi, On the ideal boundaries of abstract Riemann surfaces, Osaka Math. J. 10 (1958), 83-102. MR 20 \#3272.

4. L. Sario and M. Nakai, Classification theory of Riemann surfaces, Die Grundlehren der math. Wissenschaften, Band 164, Springer-Verlag, New York and Berlin, 1970. MR 41 \#8660.

5. J. L. Schiff, Harmonic null sets and the Painlevé theorem, Proc. Amer. Math. Soc. 43 (1974), $171-172$.

Department of Mathematics, University of Auckland, Auckland, New Zealand

Current address: Department of Mathematics, University of California, Los Angeles, California 90024 